\title{
Laser tuning in AIN single crystals
}

\author{
Yanming Zhu, Wei Zheng ${ }^{*}$, Siqi Zhu, Ying Ding, Lemin Jia and Feng Huang
}

In the fields of trace gas detection, electric field detection, visible optical communication, and beyond, laser tuning technology plays an increasingly important role, as shown in Fig. S1. Following the miniaturization of optoelectronic devices, it is desirable to integrate a large number of lasers with different wavelengths into one chip [1]. However, the dynamic wavelength allocation efficiency of fixedwavelength laser is quite low, which greatly limits the monolithic integration of optoelectronics systems. Therefore, the development of laser wavelength tuning technology is urgent and crucial to the efficiency of optoelectronics [2-7]. Raman scattering is a physical phenomenon that can be explained by mature normal form $[8,9]$. However, most studies only pay attention to the fact that Raman spectrum is a non-destructive material characterization method with fingerprint identification characteristics, while its essential feature of inelastic scattering is always ignored [10]. The natural frequency shift characteristics indicate the possibility of Raman scattering as a feasible method to realize laser wavelength tuning [11-13].

An important prerequisite for achieving laser wavelength tuning by the Raman effect is the scattering light with polarization characteristics dependent on laser medium [14]. For isotropic optical crystals, the Raman scattering signal is often accompanied by depolarization phenomenon. Thus, optical crystals with strong anisotropy may be an ideal carrier for laser wavelength tuning. Here, we adopted wurtzite aluminum nitride (AlN) as the laser medium. As a mature binary semiconductor, AlN has a relatively simple phonon dispersion relationship compared with other multicomponent systems, which directly determines a "clean" Raman spectrum [15-18]. In addition, high-quality and large-size AlN single crystals (SCs) with different crystal planes have been fabricated via physical vapor transport (PVT) technology $[19,20]$, which avoids the interference of Raman signals from other crystal planes.
In this letter, we discovered that when the polarization direction of incident light is along the $c$-axis of AlN SC, an "extinction phenomenon" will appear in $\mathrm{E}_{1}(\mathrm{TO})$ and $\mathrm{E}_{2}^{2}$ modes, and only $\mathrm{A}_{1}(\mathrm{TO})$ mode can be observed. In this situation, the scattering light corresponding to $A_{1}(T O)$ mode shows perfect linear polarization characteristics with a degree of polarization $\left(\left(I_{\|}-I_{\perp}\right) /\left(I_{\|}+I_{\perp}\right)\right)$ approaching 1. This phenomenon indicates that AlN can realize laser frequency shift with the help of Raman scattering. In addition, based on the theory that phonon frequency is limited by temperature, an ultra-high precision laser wavelength tuning $\left(0.0006 \mathrm{~nm} \mathrm{~K}^{-1}\right)$ has been realized by controlling the temperature $[21,22]$. Our experimental results indicate that wurtzite AlN with high thermal conductivity and high stability may be an ideal carrier for laser wavelength tuning [23]. Theoretically speaking, changing laser medium can achieve arbitrary wavelength tuning, which may help with the development of integrated sensing field.

Here, we adopted backscattering geometry to collect the angle-resolved polarization Raman signal of $\mathrm{m}$-plane AlN SC with Renishaw Raman spectrometer (inVia Reflex). The measurement geometry is shown in Fig. 1a [24-34], where a polarizer with fixed polarization direction was placed in the collecting optical path to ensure that the polarization direction of scattering light was strictly consistent with that of pump light. In order to avoid the influences of pump light absorption and resonance Raman scattering on the intensity of scattering light, 488-nm Ar+ gas laser was used as pump light and focused on the AlN surface through a $50 \times$ quartz lens [35-39]. Meanwhile, in order to smooth the Raman signal and prevent the sample from being damaged, exposure time and integration time were set to $1 \mathrm{~s}$ and 10 times, respectively.

Previously, we have reported an angle-resolved polarization Raman spectra of AlN microwires with a diameter of $200 \mu \mathrm{m}$ under the excitation of a 532-nm laser, in

\footnotetext{
State Key Laboratory of Optoelectronic Materials and Technologies, School of Materials, Sun Yat-sen University, Guangzhou 510275, China

* Corresponding author (email: zhengw37@mail.sysu.edu.cn)
} 
a

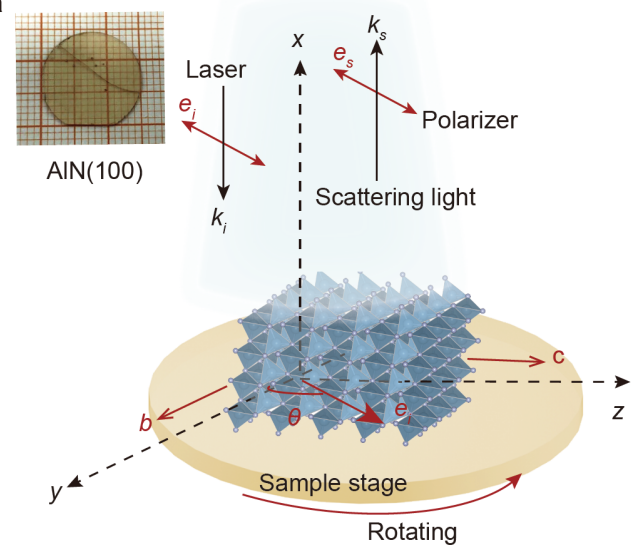

b

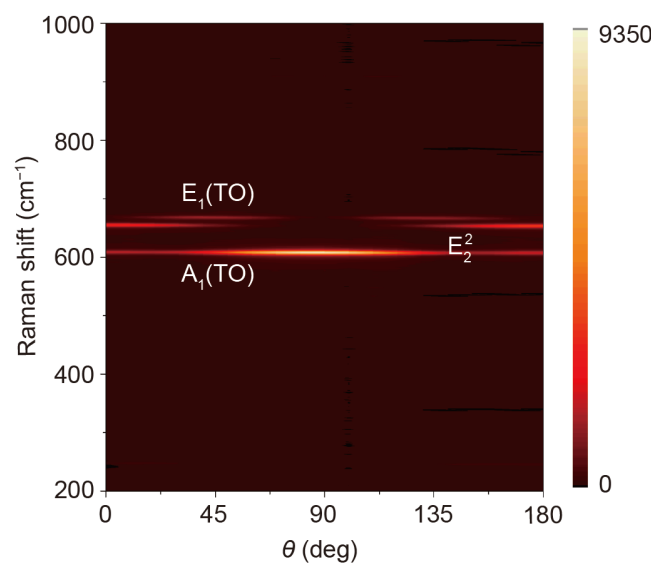

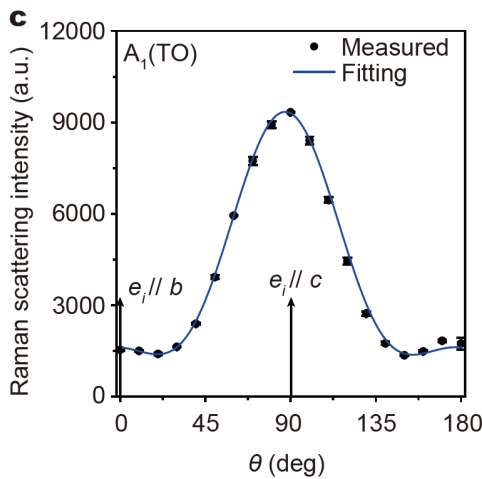
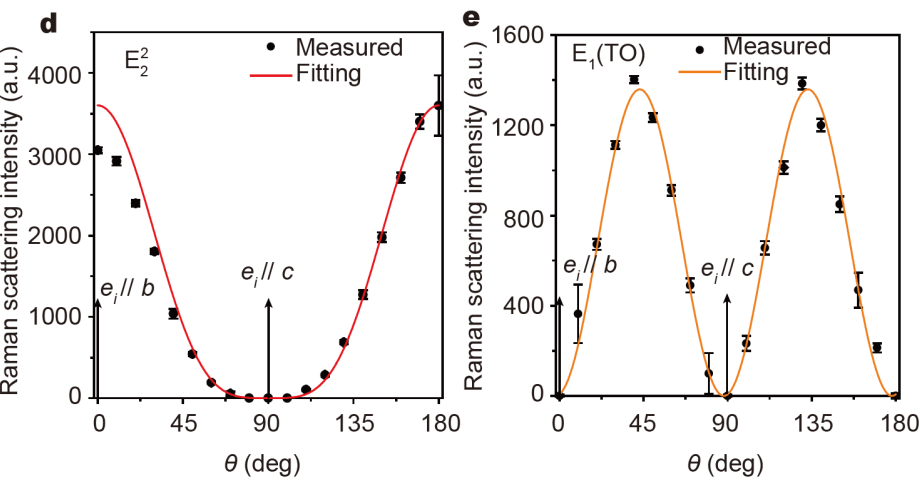

Figure 1 Angle-resolved polarization Raman spectra of m-plane AlN. (a) Backscattering polarization Raman measurement geometry. The illustration is the optical photograph of m-plane AlN SC. (b) Angle-resolved polarization Raman spectrum of m-plane AlN SC. (c-e) Raman scattering intensities of $\mathrm{A}_{1}(\mathrm{TO}), \mathrm{E}_{1}(\mathrm{TO})$ and $\mathrm{E}_{2}^{2}$ modes under different rotation angles.

which the information about its Raman tensor with a strong Raman scattering anisotropy was obtained $[20,40]$. However, laser wavelength tuning achieved by Raman scattering has a higher and stricter requirement on both the quality and flatness of crystal surface. Thus, an AlN SC with larger area and higher quality was used as the measured sample in our research. The only XRD diffraction peak [100] found in Fig. S2 indicates its perfect crystal orientation. The illustration in Fig. 1a is the optical photograph of a m-plane AlN SC with a diameter of $15 \mathrm{~mm}$ [41].

Fig. $1 \mathrm{~b}$ is the angle-resolved polarization Raman spectrum of m-plane AlN SC, which directly presents the Raman scattering intensities of three vibration modes $\left(\mathrm{A}_{1}\right.$ (TO) $\left(607.87 \mathrm{~cm}^{-1}\right), \quad \mathrm{E}_{1}$ (TO) $\left(665.87 \mathrm{~cm}^{-1}\right)$ and $\mathrm{E}_{2}^{2}$ $\left.\left(653.40 \mathrm{~cm}^{-1}\right)\right)$ under different rotation angles, of which the result is in accordance with that obtained by a $532-\mathrm{nm}$ laser $[20,40]$. From the angle-resolved polarization Raman spectrum, it can be clearly observed that when the polarization direction of pump light is along the $c$-axis of AlN, $E_{1}(T O)$ and $E_{2}^{2}$ modes will disappear, and only $A_{1}$
(TO) mode can be found with its intensity up to the maximum. The vibration laws of these modes follow the Raman selection rule, that is $I \sim\left|e_{s} \cdot R \cdot e_{i}\right|^{2}$, where $e_{s}$ and $e_{i}$ denote the polarization direction vectors of pump and scattering light, $R$ is the Raman tensor of certain phonon mode [9]. Based on the results of previous studies on the Raman polarization of AlN SC $[20,40]$, the Raman scattering intensities of different vibration modes under different rotation angles can be obtained as follows

$I_{\mathrm{A}_{1}(\mathrm{TO})}(\theta) \sim|a|^{2} \cos ^{4} \theta+|b|^{2} \sin ^{4} \theta$

$+\frac{1}{2}|a||b| \sin ^{2}(2 \theta) \cos \left(\varphi_{a}-\varphi_{b}\right)$,

$I_{\mathrm{E}_{1}(\mathrm{TO})}(\theta) \sim \frac{|c|^{2}}{2} \sin ^{2}(2 \theta)$,

$I_{\mathrm{E}_{2}^{2}}(\theta) \sim|d|^{2} \cos ^{4} \theta$,

where $a, b, c$ and $d$ are Raman tensor elements, $\varphi_{a}$ and $\varphi_{b}$ denote the complex phases of corresponding Raman tensor elements $a$ and $b$, and $\theta$ is marked as the angle between the polarization direction vector of pump light 
and the $b$-axis of AlN. Fig. 1c-e present the Raman scattering intensities of $A_{1}(T O), E_{1}(T O)$, and $E_{2}^{2}$ modes under different rotation angles and the fitting results based on Equation (1). For bulk materials, it is difficult to determine the crystal orientation through the angle-resolved polarization Raman spectrum of $\mathrm{A}_{1}(\mathrm{TO})$ mode. According to Raman selection rule, when the polarization direction of incident light is parallel to the $c$-axis of $\mathrm{m}$ plane AlN single crystal, the scatting light corresponding to $E_{2}^{2}$ mode will disappear. Returning to the results obtained from the angle-resolved polarization Raman spectrum of m-plane AlN, it is clear that when the sample is rotated by $90^{\circ}$, the polarization direction of incident light will be parallel to the $c$-axis.

As described above on the Raman scattering intensities of $\mathrm{A}_{1}(\mathrm{TO}), \mathrm{E}_{1}(\mathrm{TO})$ and $\mathrm{E}_{2}^{2}$ vibration modes, an "extinction phenomenon" can be observed when the rotation angle $\theta$ is equal to $90^{\circ}$. At this moment, a scattering light with specific wavelength can be obtained. According to the vibration frequency at $607.87 \mathrm{~cm}^{-1}$ of $A_{1}(T O)$ mode at room temperature, a corresponding wavelength of scattering light at $502.92 \mathrm{~nm}$ can be obtained. In order to verify the polarization characteristics of scattering light, we designed a specific measurement geometry as shown in Fig. 2a [31]. There are two procedures. Firstly, fixing the polarization direction of pump light parallel to the $c$ axis of AlN; secondly, changing the polarization orientation of the polarizer placed in the collection optical path to verify the depolarization characteristics of scattering light. Results are shown in Fig. 2b, that is, when the polarization direction of polarizer is along the $c$-axis of AlN, a strong scattering light can be observed, which is absolutely different from the situation when the polarization direction of polarizer is along the $b$-axis, in which $\mathrm{A}_{1}(\mathrm{TO})$ mode seems to be forbidden. This phenomenon is also consistent with the description of Raman selection rule. It is explicit that there is no depolarization phenomenon observed in the $A_{1}(\mathrm{TO})$ mode when the polarization direction of pump light is along the $c$-axis of AlN, which directly indicates that the scattering light with a
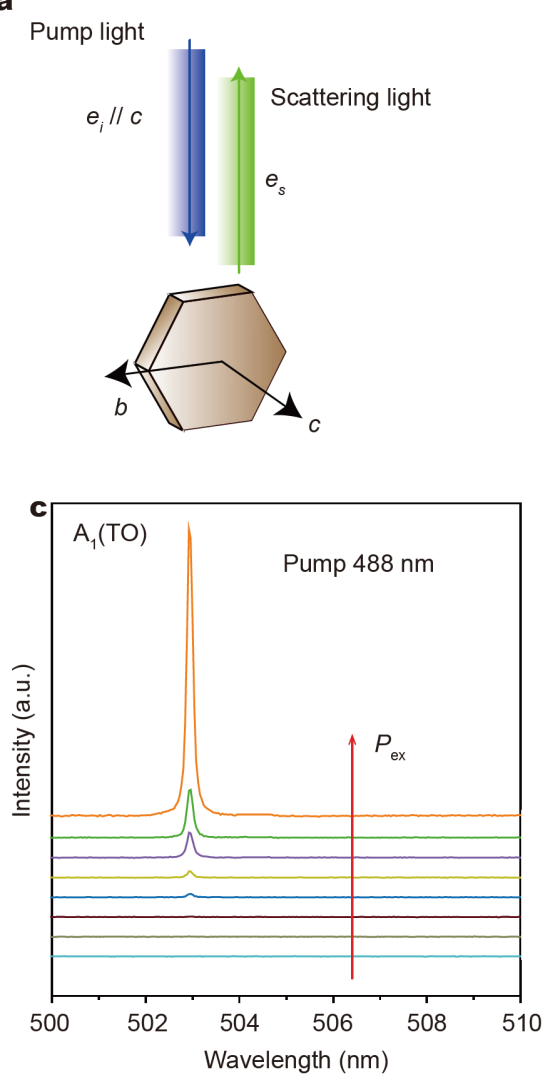

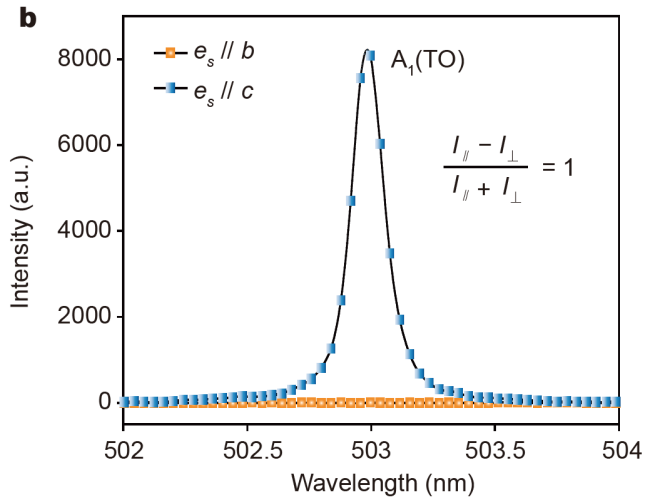

d

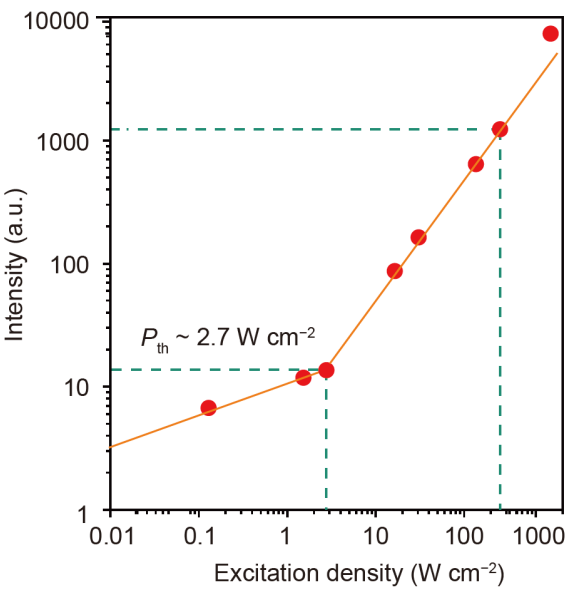

Figure 2 Verification of polarization characteristics of scattering light. (a) Schematic diagram of laser wavelength tuning. (b) Output light spectrum when the polarization direction of scattering light is parallel to the $b$-axis and $c$-axis of AlN SC, respectively. (c, d) Output light spectra and intensities under the pump light with different excitation densities. 
a wavelength of $502.92 \mathrm{~nm}$ has apparent liner polarization characteristics.

We also measured the intensities of scattering light excited by a 488-nm laser with different excitation densities. In order to measure the optical power density, we used an optical power meter whose model is NOVA II PD300 UV. Measured results are shown in Fig. 2c. With the decrease of excitation density, the intensity of scattering light also reduces rapidly. Then, we extracted the light intensities of $\mathrm{A}_{1}(\mathrm{TO})$ mode under the pump light with different excitation densities, whose results are plotted in Fig. 2d. Obviously, there is a threshold excitation density $P_{\text {th }}$, that is, only when the excitation density of 488-nm pump light is larger than $P_{\text {th }}$, can $\mathrm{A}_{1}$ (TO) mode be excited.

We measured the output light spectra of $\mathrm{A}_{1}(\mathrm{TO})$ mode at different temperatures, and the results are plotted in Fig. 3. During the measurement, the polarization direction of pump light is always parallel to the $c$-axis of AlN SC. We normalized the output light spectra at different temperatures. Then we extracted the wavelengths and FWHMs of output light at different temperatures, and the extraction results are shown in Fig. $3 c$ and d. For the wavelength, it can be observed that the wavelength of scattering light will redshift with the decrease of temperature, that is, its wavelength increases from $502.90 \mathrm{~nm}$ at $420 \mathrm{~K}$ to $503.05 \mathrm{~nm}$ at $80 \mathrm{~K}$. Besides, its change trend is also different in different temperature ranges. When the temperature of sample is higher than $250 \mathrm{~K}$, its wavelength is almost linearly redshift with an accuracy of $0.0006 \mathrm{~nm} \mathrm{~K}^{-1}$ as the temperature decreases; when the temperature is lower than $250 \mathrm{~K}$, its wavelength will increase with the decrease of temperature in a nonlinear law. For the FWHM, it can be found that the scattering light has a quite narrow FWHM, which decreases in an almost linear way from $0.17 \mathrm{~nm}$ at $420 \mathrm{~K}$ to $0.11 \mathrm{~nm}$ at $80 \mathrm{~K}$. This also suggests that Raman scattering light has a great monochromaticity close to that of commercial lasers.

In this letter, we realized wavelength modulation in $\mathrm{m}$ plane AIN SC by means of Raman scattering effect. Based on the Raman selection rule, when an "extinction phenomenon" appears in $\mathrm{E}_{1}(\mathrm{TO})$ and $\mathrm{E}_{2}^{2}$ vibration modes, only $\mathrm{A}_{1}(\mathrm{TO})$ mode can still be observed. With the help of
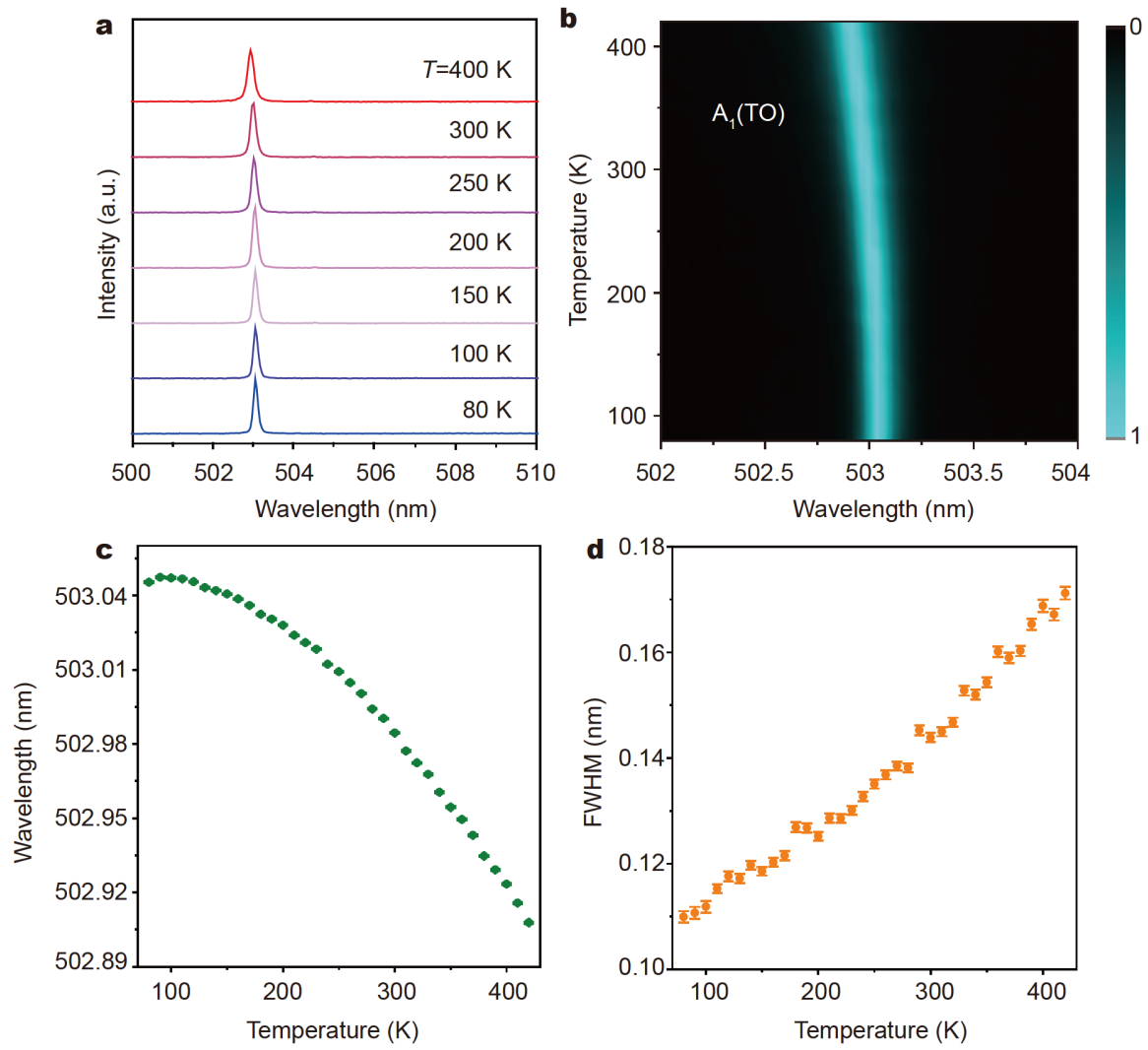

Figure 3 Temperature effect of output light. (a) Output light spectra under different temperatures. (b) Normalized output light spectra under different temperatures. (c, d) Extracted wavelengths and FWHMs of output light under different temperatures. 
a specific optical measurement geometry, we confirm that the output light corresponding to $\mathrm{A}_{1}(\mathrm{TO})$ mode has perfect liner polarization characteristics with a degree of polarization approaching 1 . Besides, the extremely narrow FWHM indicates that Raman scattering light also has great monochromaticity. Based on these experimental results, it can be expected that Raman scattering effect may be a feasible method to realize laser wavelength tuning. What's more, based on the theory that phonon frequency is limited by the temperature, laser wavelength tuning with an accuracy of $0.0006 \mathrm{~nm} \mathrm{~K}^{-1}$ was achieved via temperature regulation. Theoretically speaking, changing the laser medium can realize arbitrary wavelength tuning, which is an important reference to achieving monolithic multi-channel coherent optical communication.

Received 5 March 2021; accepted 23 April 2021;

published online 24 June 2021

1 La Monica S, Maiello G, Ferrari A, et al. Progress in the field of integrated optoelectronics based on porous silicon. Thin Solid Films, 1997, 297: 265-267

2 Ulvila V, Vainio M. Diode-laser-pumped continuous-wave optical parametric oscillator with a large mid-infrared tuning range. Optics Commun, 2019, 439: 99-102

3 Yao T, Chen Y, Zhang Y, et al. All-fiberized cascaded random Raman fiber laser with high spectral purity based on filtering feedback. Appl Opt, 2019, 58: 9728-9733

4 Willner AE, Syang-Myau Hwan AE. Transmission of many WDM channels through a cascade of EDFA's in long-distance links and ring networks. J Lightwave Technol, 1995, 13: 802-816

5 Maiman TH. Stimulated optical radiation in ruby. Nature, 1960, 187: 493-494

6 Li S, Zhang Y, Yang W, et al. 2D perovskite $\mathrm{Sr}_{2} \mathrm{Nb}_{3} \mathrm{O}_{10}$ for highperformance UV photodetectors. Adv Mater, 2020, 32: 1905443

$7 \mathrm{Xu} \mathrm{X}$, Chen J, Cai S, et al. A real-time wearable UV-radiation monitor based on a high-performance $\mathrm{p}-\mathrm{CuZnS} / \mathrm{n}-\mathrm{TiO}_{2}$ photodetector. Adv Mater, 2018, 30: 1803165

8 Loudon R. The Raman effect in crystals. Adv Phys, 2001, 50: 813864

9 Cardona M. Resonance phenomena. In: Cardona M, Güntherodt G (eds). Light Scattering in Solids II: Basic Concepts and Instrumentation. Berlin, Heidelberg: Springer Berlin Heidelberg, 1982: 19-178

10 Raman CV. A new radiation. Indian J Phys, 1928, 2: 387-398

11 Lewis SAE, Chernikov SV, Taylor JR. Gain and saturation characteristics of dual-wavelength-pumped silica-fibre Raman amplifiers. Electron Lett, 1999, 35: 1178-1179

12 Al-Alimi AW, Cholan NA, Yaacob MH, et al. Wideband multiwavelength output generation based on cascaded four-wave mixing in distributed Raman amplifier utilizing a Fabry-Pérot laser diode. Optics Laser Tech, 2017, 93: 87-91

13 Zheng W, Li F, Li G, et al. Laser tuning in van der Waals crystals. ACS Nano, 2018, 12: 2001-2007

14 Porto SPS, Giordmaine JA, Damen TC. Depolarization of Raman scattering in calcite. Phys Rev, 1966, 147: 608-611
15 McNeil LE, Grimsditch M, French RH. Vibrational spectroscopy of aluminum nitride. J Am Ceramic Soc, 1993, 76: 1132-1136

16 Carlone C, Lakin KM, Shanks HR. Optical phonons of aluminum nitride. J Appl Phys, 1984, 55: 4010-4014

17 Li X, Zhou C, Jiang G, et al. Raman analysis of aluminum nitride at high temperature. Mater Charact, 2006, 57: 105-110

18 Bungaro C, Rapcewicz K, Bernholc J. Ab initio phonon dispersions of wurtzite AlN, GaN, and InN. Phys Rev B, 2000, 61: 6720-6725

19 Wang G, Zhang L, Wang Y, et al. Growth and stress analysis of spontaneous nucleation $c$-plane bulk AlN crystals by a PVT method. Cryst Res Tech, 2020, 55: 2000118

20 Zheng W, Zheng RS, Wu HL, et al. Strongly anisotropic behavior of $\mathrm{A}_{1}(\mathrm{TO})$ phonon mode in bulk AlN. J Alloys Compd, 2014, 584: 374-376

21 Zhang Z, Zheng W, Wang W, et al. Anisotropic temperaturedependence of optical phonons in layered $\mathrm{PbI}_{2}$. J Raman Spectrosc, 2018, 49: 775-779

$22 \mathrm{He} \mathrm{H}$, Zhang Z. Decay mechanism of optical phonons in $\gamma$-CuI. AIP Adv, 2019, 9: 055104

23 Zinkle SJ, Skuratov VA, Hoelzer DT. On the conflicting roles of ionizing radiation in ceramics. Nucl Instruments Methods Phys Res Sect B-Beam Interactions Mater Atoms, 2002, 191: 758-766

24 Ding Y, Zheng W, Lin Z, et al. Raman tensor of layered $\mathrm{WS}_{2}$. Sci China Mater, 2020, 63: 1848-1854

25 Ding $\mathrm{Y}$, Zheng W, Jin M, et al. Raman tensor of layered $\mathrm{MoS}_{2}$. Opt Lett, 2020, 45: 1313-1316

26 Jin $\mathrm{M}$, Zheng W, Ding Y, et al. Raman tensor of $\mathrm{WSe}_{2}$ via angleresolved polarized Raman spectroscopy. J Phys Chem C, 2019, 123: 29337-29342

27 Ribeiro HB, Pimenta MA, de Matos CJS. Raman spectroscopy in black phosphorus. J Raman Spectrosc, 2018, 49: 76-90

28 Zhu Y, Zheng W, Wang W, et al. Raman tensor of layered black arsenic. J Raman Spectrosc, 2020, 51: 1324-1330

29 Zhu Y, Zheng W, Wang W, et al. Raman tensor of layered black phosphorus. PhotoniX, 2020, 1: 17

30 Li T, Luo W, Kitadai $\mathrm{H}$, et al. Probing the domain architecture in 2D a- $\mathrm{Mo}_{2} \mathrm{C}$ via polarized Raman spectroscopy. Adv Mater, 2019, 31: 1807160

31 Huang S, Tatsumi Y, Ling X, et al. In-plane optical anisotropy of layered gallium telluride. ACS Nano, 2016, 10: 8964-8972

32 Liu XL, Zhang X, Lin ML, et al. Different angle-resolved polarization configurations of Raman spectroscopy: A case on the basal and edge plane of two-dimensional materials. Chin Phys B, 2017, 26: 067802

33 Ding Y, Zheng W, Zhu Y, et al. Raman tensor of layered Td-WTe ${ }_{2}$. J Phys Chem C, 2020, 124: 16596-16603

34 Ding Y, Zheng W, Lu X, et al. Raman tensor of layered $\mathrm{SnS}_{2}$. J Phys Chem Lett, 2020, 11: 10094-10099

35 Oto T, Banal RG, Kataoka $\mathrm{K}$, et al. $100 \mathrm{~mW}$ deep-ultraviolet emission from aluminium-nitride-based quantum wells pumped by an electron beam. Nat Photon, 2010, 4: 767-770

36 Taniyasu Y, Kasu M, Makimoto T. An aluminium nitride lightemitting diode with a wavelength of 210 nanometres. Nature, 2006, 441: $325-328$

37 Zhu Y, Lin R, Zheng W, et al. Near vacuum-ultraviolet aperiodic oscillation emission of AlN films. Sci Bull, 2020, 65: 827-831

38 Zheng W, Zhu Y, Li F, et al. Raman spectroscopy regulation in van der Waals crystals. Photon Res, 2018, 6: 991-995

39 Jin S, Zhang Y, Fan F, et al. Deep UV resonance Raman spectroscopic study on electron-phonon coupling in hexagonal III-nitride 
wide bandgap semiconductors. J Raman Spectrosc, 2016, 47: 884887

40 Zheng W, Zheng R, Huang F, et al. Raman tensor of AlN bulk single crystal. Photon Res, 2015, 3: 38-43

41 Lin R, Zheng W, Chen L, et al. X-ray radiation excited ultralong $(>20,000$ seconds) intrinsic phosphorescence in aluminum nitride single-crystal scintillators. Nat Commun, 2020, 11: 4351

Acknowledgements This work was financially supported by the National Natural Science Foundation of China (91833301 and 61604178).

Author contributions Zhu Y designed the experimental details. Zheng $\mathrm{W}$ directed this program. Zhu Y, Zhu S, Ding Y and Jia L led the experimental work. Huang F participated in the discussion. Zhu Y contributed to the writing of the manuscript.

Conflict of interest The authors declare that they have no conflict of interest.

Supplementary information Supplementary information and supporting data are available in the online version of the paper.

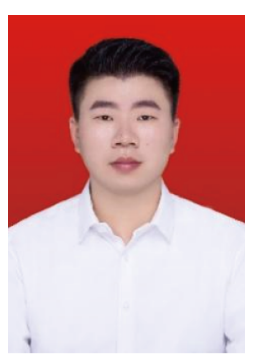

Yanming Zhu received his bachelor degree in physics at Zhejiang Normal University in 2016, and now he is studying for a $\mathrm{PhD}$ degree of materials physics and chemistry at the School of Materials of Sun Yat-sen University, with the research focusing on the fluorescence characteristics and Raman spectra of wide bandgap semiconductor and low-dimensional materials.

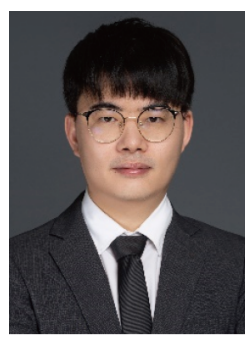

Wei Zheng received his $\mathrm{PhD}$ degree from Shenzhen University in 2014. His research interest focuses on semiconductor-based vacuumultraviolet $(10-200 \mathrm{~nm})$ photodetectors and condensed matter physics in ultra-wide bandgap semiconductors.

\section{AlN单晶中的激光调谐}

朱燕明, 郑伟 ${ }^{*}$, 朱思琪, 丁莹, 贾乐敏, 黄丰

摘要 激光波长调谐对于在压力检测、强电场监测、痕量气体探 测以及可见光通讯领域中提高动态波长分配效率起着重要作用. 拉曼散射是一种具有频移特性的非线性光学效应, 这表明拉曼散 射可能是一种实现激光波长调谐的可行方法. 在本文中, 我们发现 在特定的测试结构下, 氮化铝的 $A_{1}(T O)$ 模式对应的散射光具有线 偏振特性. 此外, 通过温度调制, 我们在氮化铝中实现了高精度的 散射光波长调谐. 基于实验结果, 具有高热导率和高稳定性的氮化 铝可能是一种实现激光波长调谐的良好载体. 